\title{
339 - A psychological profile of near-centenarians and centenarians with and without dementia from the Sydney Centenarian Study
}

Authors

Adrian Cheng ${ }^{1,2}$, Yvonne Leung ${ }^{1,5}$, John Crawford ${ }^{1}$, Fleur Harrison ${ }^{1,2}$, Perminder Sachdev ${ }^{1,4}$, Henry Brodaty ${ }^{1,2,3}$

1. Centre for Healthy Brain Ageing, School of Psychiatry, University of New South Wales, Sydney, Australia

2. Dementia Collaborative Research Centre - Assessment and Better Care, School of Psychiatry, University of New South Wales, Sydney, Australia

3. Academic Department for Old Age Psychiatry, Prince of Wales Hospital, Randwick, Australia

4. Neuropsychiatric Institute, Prince of Wales Hospital, Randwick, Australia

5. School of Psychology, University of Nottingham Malaysia, Malaysia

Background: Studying those who have achieved exceptional longevity can provide a model of successful ageing, however current research remains limited. Previous work on centenarians has primarily focused on depression and anxiety; life satisfaction remains understudied.

Objectives: To compare the psychological profile of near-centenarians (95-99) and centenarians (100+) with and without dementia. To compare the psychological distress and life satisfaction in nearcentenarians and centenarians without dementia with younger age groups. To identify the risk and protective factors of psychological distress and life satisfaction in near-centenarians and centenarians without dementia.

Methods: The Sydney Centenarian Study (SCS) collected data from 343 participants aged 95 years and older, of whom 119 had dementia. Psychological distress was assessed using the Kessler Psychological Distress Scale (K10). Life satisfaction was measured using the Satisfaction with Life Scale (SWLS). Persons aged 70-90 years from the Sydney Memory and Ageing Study (MAS) were used as a cross-sectional comparison group, for which dementia was an exclusion. Multiple linear regressions were undertaken to investigate the predictors for psychological distress and life satisfaction in SCS.

Findings: There was no significant difference in K10 or SWLS score between SCS participants with and without dementia. SCS participants without dementia reported significantly higher levels of psychological distress $(15.3,13.4, \mathrm{t}=3.869, \mathrm{p}<0.001)$ and life satisfaction $(6.0,5.6, \mathrm{t}=5.835, \mathrm{p}<0.001)$ compared to cognitively intact younger age groups in MAS. In SCS, a greater number of psychotropic medications and less contact with friends and family were associated with higher psychological distress. Higher scores on the Mini-Mental State Examination and greater contact with friends and family were associated with higher life satisfaction.

Conclusions: Psychological health was similar in near-centenarians and centenarians whether or not they had dementia. Although near-centenarians and centenarians without dementia demonstrated higher levels of psychological distress in the past 4 weeks than younger age groups, their satisfaction with life was higher. Social support and cognition may be protective factors against poor psychological health and promote greater life satisfaction. Factors identified as associated with psychological distress and life satisfaction may be targets for interventions to maintain good psychological health in this vulnerable population. 